\title{
Automated Quantification of Non-Calcified Coronary Plaques in Cardiac CT Angiographic Imagery
}

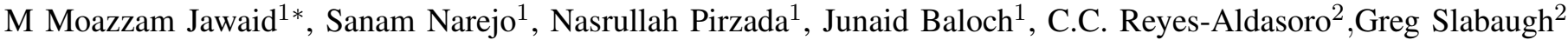 \\ ${ }^{1}$ Faculty of Electrical \& Electronic Engg. \\ Mehran University of Engineering \& Technology, Jamshoro 76062 \\ ${ }^{2}$ Deptt. of Electrical \& Electronic Engg. \\ City University London, EC1V OHB
}

\begin{abstract}
The high mortality rate associated with coronary heart disease (CHD) has driven intensive research in cardiac image analysis. The advent of computed tomography angiography (CTA) has turned non-invasive diagnosis of cardiovascular anomalies into reality as calcified coronary plaques can be easily identified due to high intensity values. However, detection and quantification of the non-calcified plaques in CTA is still a challenging problem because of their lower intensity values, which are often similar to the nearby blood and muscle tissues. In this work, we propose Bayesian posterior based model for precise quantification of the non-calcified plaques in CTA imagery. The only indicator of non-calcified plaques in CTA is relatively lower intensity. Hence, we exploited intensity variations to discriminate voxels into lumen and plaque classes. Based on the normal coronary segments, we computed the vessel-wall thickness in first step. In the subsequent step, we removed vessel wall from the segmented tree and employed Gaussian Mixture Model to compute optimal distribution parameters. In the final step, distribution parameters were employed in Bayesian posterior model to classify voxels into lumen or plaque. A total of 18 CTA volumes were analyzed in this work using two different approaches. According to the experimental results, mean Jaccard overlap is around $88 \%$ with respect to the manual expert. In terms of sensitivity, specificity and accuracy, the proposed method achieves $84.13 \%$ ,79.15\% and $\mathbf{8 2 . 0 2 \%}$ success, respectively. Conclusion: According to the experimental results, it is shown that the proposed plaque quantification method achieves accuracy equivalent to human experts.
\end{abstract}

Keywords-Coronary segmentation; non-calcified plaques; vascular quantification; coronary wall analysis

\section{INTRODUCTION}

Coronary heart disease (CHD) is related to the accumulation of fatty materials (also termed as coronary plaques) inside coronary arteries. The recent statistics of the National Health Services, United Kingdom [2] reveals that over 2.3 million people in the United Kingdom suffer from CHD where the annual death toll is approximately 73,000 (an average of one death every seven minutes). The substantial levels of growing morbidity and mortality have led to a intensified interest in new techniques for detecting coronary abnormalities to potentially avoid worst events [1], [2].

The recent advancements in non-invasive imaging have improved the diagnostic accuracy in terms of high temporal and spatial resolution [3]; however, detection and quantification of non-calcified plaques in CTA is still a challenging problem. Clinically, the non-calcified plaques have been established as the most important indicator of acute coronary syndromes due to their fragile nature [4]. The risk of sudden rupture has made soft plaques threatening in clinical context, i.e. for many individuals, sudden death becomes the first sign of soft plaque in contrast to the calcified plaques which often lead to disease symptoms at early stages. It should be noted that calcified plaques can be identified easily in a CTA image based on the high intensity value, consequently numerous methods have been reported with a reasonable quantification accuracy [5][8]; however, non-calcified plaque requires more sophisticated phenomenon. In context of the flow of paper, we start with relevant literature and CTA data specification. Subsequently, we explain the plaque quantification methodology which is followed with the Results section. The lumen - plaque quantification results are provided in the Results section using statistical metrics of sensitivity, specificity and accuracy, with respect to manual experts.

\section{RELATED WORK}

Non-calcified plaque detection and quantification in CTA has been a challenging problem; hence, there is a little literature [9]-[12], [14] published addressing automatic segmentation, out of which the majority have been clinical pilot studies or generic anomaly detection techniques. The use of machine learning in soft plaque detection was first reported by Wei et al. [10] where a linear discriminant analysis (LDA) was used to reduce the false positives in a set of 120 preselected soft plaque candidates. Accordingly, the detection accuracies reported were $94 \%$ and $79 \%$, respectively for the calcified and non-calcified plaques, along with a high number of false positives. Another interesting method for the automatic detection of vascular abnormalities was proposed by Zuluaga et al. [13]. In this work, an unsupervised SVM model trained on normal cross sections was used to detect the outliers i.e. the cross sections which violate the intensity pattern of normal class. The authors reported promising results for 9 clinical CTAs with NCP detection accuracy of $79.62 \%$, however; the precise quantification was not performed in this work. Similarly, the detection methods were reported by Renard and Yang [14], Lankton et al. [11], Li et al. [15]; however, the precise quantification has not been reported frequently.

In context of the non-calcified plaque quantification, a number of algorithms [16], [17], [34], [35] have been proposed in recent years with a motive of correlating CTA based plaque quantification with intra-vascular ultrasound (IVUS) measurements. Athanasiou et al. [35] employed 4-class Gaussian Mixture Model to identify respective classes namely lumen, 
calcified plaque, non-calcified plaques and the background. Accordingly, the paper reported efficiency over the existing literature and a good correlation with IVUS measurements; however, the blooming effect of calcified plaque resulted in relatively low agreement for calcified plaque volume. Moreover, the vessel wall analysis was not performed explicitly, which is very crucial in context of the non-calcified plaques because of two-way vessel remodelling.

In addition, a number of studies [16], [18]-[23], [34], [36][39] have been reported in context of the non-calcified plaque quantification: however, the main focus in these studies was to demonstrate the capability of CTA imaging to reflect the non-calcified plaque rather than automated quantification of non-calcified plaque in CTA. Accordingly, non-calcified plaque lesions were manually selected in the first step, and plaque quantification results were compared with respect to intravascular ultrasound analysis to establish correlation between two imaging modalities.

Our contribution in this work is an efficient methodology for quantification of the non-calcified plaques with a humanequivalent accuracy. First, we present an efficient method for the vessel wall analysis in context of the non-calcified plaques. The proposed vessel-wall analysis can be used as a standalone plaque detection method as well it serves as important step towards plaque quantification. In addition, we formulate a posterior class based plaque quantification method for voxelwise plaque quantification with a human-equivalent accuracy.

In this work, we employed clinical CTA data (a total of 16 CTA images) obtained from publicly available database of Rotterdam Coronary Artery Evaluation framework [24], [25]. The Rotterdam CTA data comes from different sources and is based on different vendors as described in [24]. The motive behind using Rotterdam data is the availability of the manual ground truth in terms of expert annotations i.e. segment- wise status (normal/abnormal) and the precise position of non-calcified plaque for the abnormal coronary segments. Based on the provided ground truth, we identified the individual coronary segments affected with non-calcified plaques as defined in Table I.

TABLE I. Non-CAlcified Plaque EFFECted SEgments in ROTTERDAM CTA DATA

\begin{tabular}{lclcc}
\hline \multirow{2}{*}{ Segment ID } & \multicolumn{4}{c}{ Plaque Specifications } \\
\cline { 2 - 5 } & Segment Type & Plaque Type & Plaque Grading & Stenosis(\%) \\
\hline DS1 seg6 & Proximal & Non-calcified & mild & 20 \\
DS2 seg6 & Proximal & Non-calcified & mild & 25 \\
DS4 seg1 & Proximal & Non-calcified & Severe & 65 \\
DS4 seg2 & Proximal & Non-calcified & Moderate & 51 \\
DS5 seg2 & Proximal & Non-calcified & Moderate & 57 \\
DS5 seg8 & Distal & Non-calcified & Moderate & 45 \\
DS7 seg2 & Proximal & Non-calcified & Severe & 71 \\
DS7 seg3 & Proximal & Non-calcified & Moderate & 41 \\
DS9 seg2 & Proximal & Non-calcified & Moderate & 51 \\
DS11 seg7 & Proximal & Non-calcified & Mild & 22 \\
DS15 seg2 & Proximal & Non-calcified & Moderate & 53 \\
DS15 seg3 & Proximal & Non-calcified & Mild & 22 \\
DS15 seg14 & Distal & Non-calcified & Moderate & 45 \\
\hline
\end{tabular}

\section{PROPOSED MODEL}

Precise segmentation of the coronary vasculature serves as first step in plaque quantification. Accordingly, we employed hybrid energy model of [26] to extract the coronary tree as

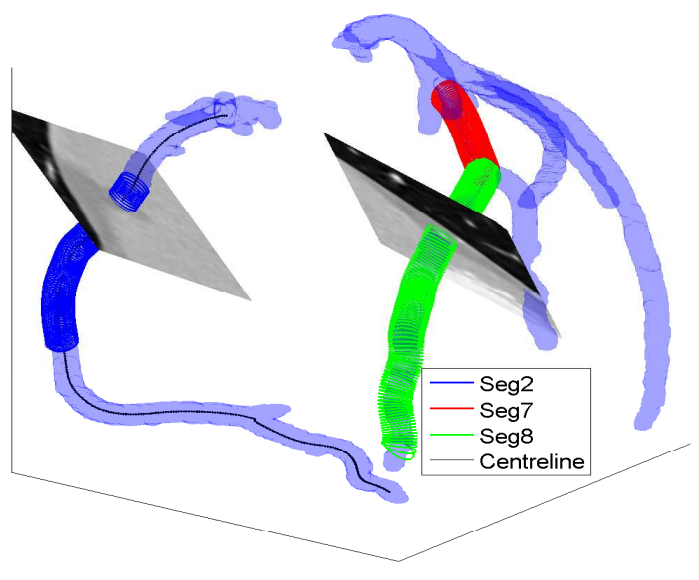

(a) DS04 seg1

Fig. 1. Segmented coronary trees with overlaid centreline and two cross sectional planes. The centreline is overlaid in black colour for the right coronary artery, whereas blue, red and green represents the curved cylindrical approximations for coronary segments numbered 2, 7 and 8 respectively.

illustrated in Fig. 1. Subsequently, radial profile based plaque detection method [27] was applied to precisely localise the plaque in different coronary segments.

\section{A. Ground Truth Construction}

In context of plaque quantification, we started with the "reference" ground truth formulation using plaque position inside respective coronary segments. Because of the ambiguous appearance in CTA imagery, the non-calcified plaque is clinically estimated by evaluating lumen deformations. Accordingly, we used the annotated lumen boundary of Rotterdam experts to derive the voxel-wise plaque ground truth. The lumen diameter variations can be observed in the mid of the vessel as shown in Fig. $2 \mathrm{a}-2 \mathrm{~b}$, indicating non-calcified plaque instance at respective locations. We approximated the ideal "plaque-free" vessel (red contours) for the plaque affected region using two "normal" cross sections (immediately before and after the plaque region) as shown in Fig. $2 c-2 d$. In the subsequent step, the annotated lumen (black contour) is subtracted from the ideal vessel (red contour) and the remaining voxels in the plaque free region are labelled as ground truth plaque voxels.

For mathematical formulation of the plaque estimation problem, we represent the coronary segment (lumen boundary annotations) using a tubular model $T_{\text {model }}\left[C P, \theta_{c s}^{\prime}\right]$, where $C P$ denotes the centreline of the segment and $\theta_{c s}^{\prime}$ defines corresponding cross-sectional information. Accordingly, complete coronary segment is represented using an $\left[N_{s}\right]$ by $[m]$ array, where $N_{s}$ represent the total number of points in segment centreline and $m$ denotes cross-sections related parameters.

The elliptical model is used to represent vascular cross sections, as Vessels are elastic bodies which can accommodate local deformations of the lumen due to changes in the blood flow and intra-luminal pressure. Such deformations cannot 

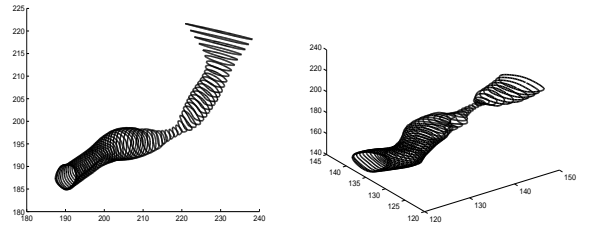

(a) DS4Seg1-lumen reduc-(b) DS7Seg2-lumen reduction

tion
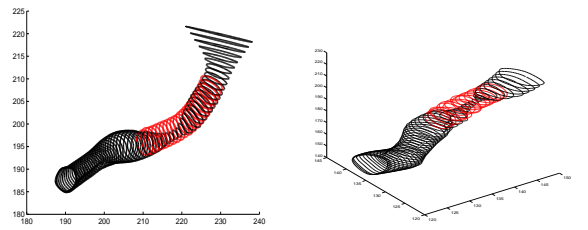

(c) DS4Seg1-ideal vessel (d) DS7Seg2-ideal vessel

Fig. 2. Lumen boundary annotations for two non-calcified plaque effected coronary segments. Black contours represent manual annotations for lumen boundary in 3D space, (red) contours define "ideal" (plaque-free) vessel boundary for the plaque effected region of the coronary segment.

be accurately represented using circular cross section model proposed in [28]-[32].

The elliptical model based representation used in this work is illustrated in Fig. 3a. Accordingly, for the $i^{\text {th }}$ point of the centreline $C P$, we define the parameter vector $\theta_{c s}^{\prime}(i)$ using ellipse as $\left[E_{x y z}(i) \approx\left\{a(i), b(i), C_{x y z}(i), R_{x y z}(i)\right\}\right]$, where $a(i)$ and $b(i)$ represent the semi axis length for major and minor axes of the current ellipse, $C_{x y z}(i)$ denotes the centre of the $i^{\text {th }}$ ellipse of segment, $R_{x y z}(i)$ defines orientation information for $i^{\text {th }}$ ellipse and $E_{x y z}(i)$ represents points on the ellipse circumference. Accordingly, the mathematical formulation (parametric representation) for a 3-dimensional ellipse is expressed by (1), where $t^{\prime}$ denotes the angular parameter varying between 0 to $2 \pi$.

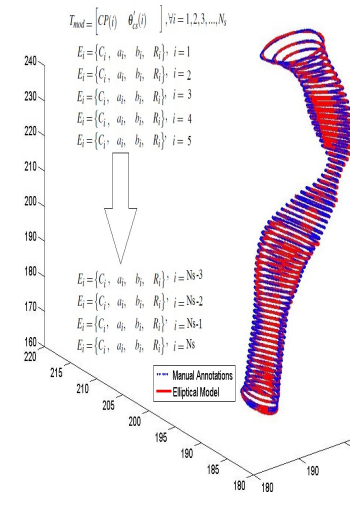

(a)

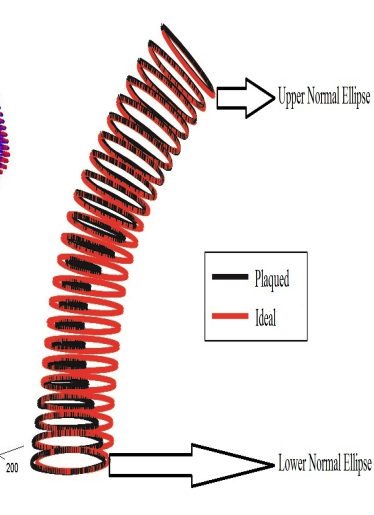

(b)
Fig. 3. Tubular model representation and estimation of ideal vessel boundary for plaque effected region of coronary segment. Black contours represent manually annotated lumen boundary in the plaque effected region, red shows the estimated ideal (plaque-free) vessel boundary based on two normal (upper and lower) cross sections.

$$
E_{x y z}=\left[\begin{array}{c}
C x \\
C y \\
C z
\end{array}\right]+R_{x y z}\left[\begin{array}{c}
a \cdot \cos \left(t_{1}\right) \\
b \cdot \sin \left(t_{1}\right) \\
0
\end{array}\right]
$$

where, $R_{x y z}=R_{1} \cdot R_{2} \cdot R_{3}$, and individual rotation values are computed as follows:

$$
\begin{aligned}
& R 1=\left[\begin{array}{ccc}
\cos (\alpha) & \sin (\alpha) & 0 \\
-\sin (\alpha) & \cos (\alpha) & 0 \\
0 & 0 & 1
\end{array}\right], \\
& R 2=\left[\begin{array}{ccc}
1 & 0 & 0 \\
0 & \cos (\beta) & \sin (\beta) \\
0 & -\sin (\beta) & \cos (\beta)
\end{array}\right], \\
& R 3=\left[\begin{array}{ccc}
\cos (\gamma) & \sin (\gamma) & 0 \\
-\sin (\gamma) & \cos (\gamma) & 0 \\
0 & 0 & 1
\end{array}\right] .
\end{aligned}
$$

Accordingly, for an ellipse based modelling of the respective coronary segment, we approximated the manually annotated lumen boundaries (3D- contours) using best fitting ellipses on respective cross sections of the coronary segment using non-linear least square fitting. After obtaining the elliptical model $T_{\text {model }}\left[C P, \theta_{c s}^{\prime}\right]$ of the coronary segment, we used two "normal" ellipses adjacent to the lesion region i.e (immediately before and after the plaque region) to derive the parameters for ideal ellipse (plaque-free vessel) through the plaque affected region as illustrated in Fig. 3b. It should be noted that, in order to model the ideal plaque-free vessel at $i^{t h}$ point of the centreline, we employed the ellipse orientation information from the current fitted ellipse i.e. $R_{x y z}(i)$, whereas the major-minor axis lengths for ideal ellipse are derived from two "normal" ellipses $E_{s}$ and $E_{e}$, which ensures that the 3D progression of vessel is tracked realistically.

$$
E_{x y z}(i)=\left\{a(i) \quad b(i), \quad C_{x y z}(i), \quad R_{x y z}(i)\right\}
$$

where $a(i)$ and $b(i)$ represent major-minor axes derived from two normal ellipses adjacent to the plaque region i.e. immediately before and after the plaque region.

After deriving the ideal ellipses for the plaque effected region, we subtracted the manually annotated lumen region which results in "reference" ground truth plaque voxels. The process of obtaining plaque ground truth is further illustrated in Fig. 4 where lumen boundary contours are used effectively in plaque identification process. It can be observed that due to the presence of a non-calcified plaque, the lumen shrinks in the proximal section and overcomes the diameter reduction as plaque region is passed. The left column of the figure represents the ideal vessel at respective cross sections of the segment, the middle column shows the manually annotated lumen and the right column represents the leftover to be interpreted as non-calcified plaque. It can be observed from the middle column that the lumen annotations are closely corroborating the plaque-free vessel for two normal contours (top and bottom row), whereas the lumen contour in middle row (plaque affected) appears significantly reduced. Likewise, the right column justifies that there exist a minimal plaque for 
two normal cross-sections, whereas the plaque effected crosssection results in a substantial amount of non-calcified plaque.

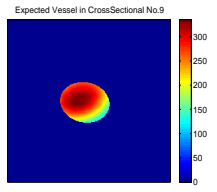

(a) Ideal vessel

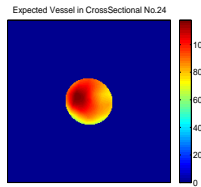

(d) Ideal vessel

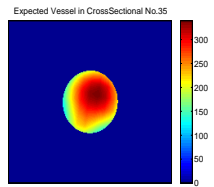

(g) Ideal vessel

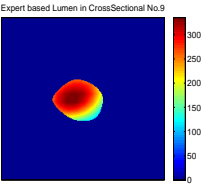

(b) Lumen

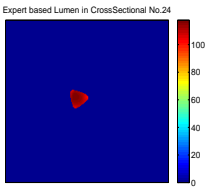

(e) Lumen

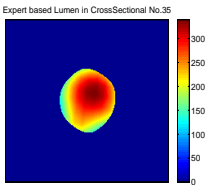

(h) Lumen (c) Plaque

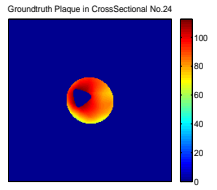

(f) Plaque

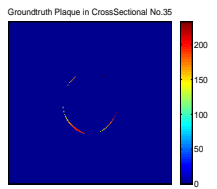

(i) Plaque

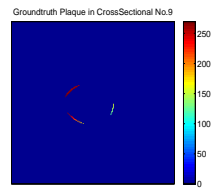

Fig. 4. The ground truth estimation for plaque-effected cross-sections using lumen boundary annotations of manual expert. The Top and bottom rows show two normal slices at the start and end of the plaque region, whereas the middle row represents a severely effected plaque cross section. The first column shows ideal vessel, middle column shows the manually annotated lumen and right column shows derived plaque.

\section{B. Vessel Wall Analysis}

The non-calcified plaque quantification algorithm is based on the assumption that the input data (vessel) comprises of two components (i.e. blood lumen and the non-calcified plaque); however, the initial segmented tree violates this basic assumption. This is due to the fact that initially segmented tree includes the vessel wall, i.e. the interface of the lumen with the background in CTA imagery. Hence, the vessel wall must be identified and removed before applying the non-calcified plaque quantification algorithm. Accordingly, we started with the segmented coronary tree and computed the vessel wall thickness for normal segments in respective $C T A_{s}$. In the subsequent step, the vessel wall is removed using ray projection based thickness metric. In the final step, wallremoved coronary segments are evaluated for the lumen and non-calcified plaques.

The wall thickness computation process starts with the cylindrical model of Fig. 1, in which a coronary segment is approximated using 6 millimeters based cylindrical model for segment approximation. Based on the fact that 6-mm represents the maximum possible expansion of coronary vessel, the background data is often included in circular approximation. Accordingly, we used three class Gaussian Mixture Model (GMM), followed with the Bayesian Posterior's computation to classify the tubular segment voxels into three classes namely the background, vessel wall and the lumen as illustrated in Fig. 5 .

Accordingly, it can be observed from the second column of the figure that background is generally well identified by

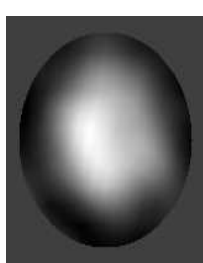

(a) image

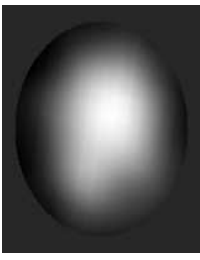

(e) image

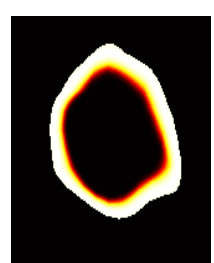

(b) back

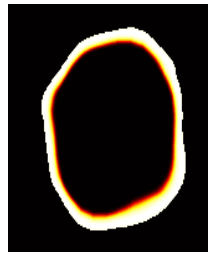

(f) back

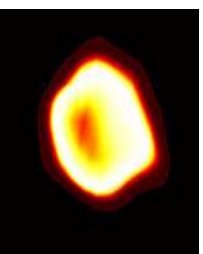

(c) wall

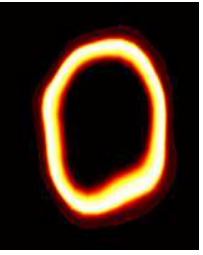

(g) wall

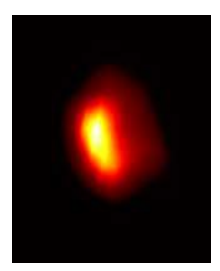

(d) Lumen

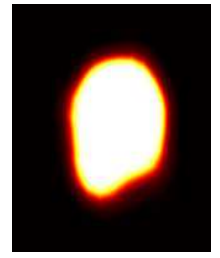

(h) Lumen
Fig. 5. Vessel wall analysis based on 3-class approximation of $6 \mathrm{~mm}$ cylindrical model of DS4 seg1. First column shows $6 \mathrm{~mm}$ region on the crosssectional plane, second column represents the background of vessel that comes inside $6 \mathrm{~mm}$, next two columns shows the vessel wall and lumen respectively. First and third row represents two normal cross-sections, whereas the middle row represents an abnormal cross section.

"class-1" as first peak of the histogram corresponds to the low intensity regions that appears dark-black in the $6 \mathrm{~mm}$ circle of first column. Likewise, "class-2" defining vessel wall is represented in the middle column in which a ring pattern circumscribing the lumen can be clearly visualized. Class-3 representing lumen is shown in column 4 of the figure where a stable pattern can be observed for normal cross sections (top and bottom row) along the length of the segment. In case of plaque effected cross-section(middle row), the 3-class approximation reflects the abnormality in terms of violation of the normal patterns for both lumen and the vessel wall. The non-calcified plaque in general assumes intensity value comparatively lower than the blood lumen and close to the myocardial tissues. Hence, our 3-class approximation assigns the existing non-calcified plaque voxels to "class-2" i.e. the vessel wall. Consequently, the vessel wall shows unexpected increase in thickness for non-calcified plaque-effected sections with a significant reduction in lumen as illustrated in Fig. 5a $-5 \mathrm{~d}$.

After identifying the vessel wall, we employed rayprojection technique to compute the wall thickness for arterial cross section as illustrated in Fig. 6a - 6c. Based on the centre of the lumen, we projected a total of 36 rays outward with an angular interval of 10 degrees and computed ray-wise thickness of the vessel wall, which is averaged to obtain the wall thickness for respective cross section. This phenomena is further illustrated using wall thickness plots for two plaque affected segments as shown in Fig. 6d - 6e. It can be observed that for both segments, the lumen (black) starts with sharp decrement and becomes stable as we move away from the aorta. Similarly, the wall thickness (red) shows a stable thickness value for normal region of the segment. However, the plaque affected region shows unexpected reduction in lumen coupled with unexpected increase in the wall thickness. Once the mean wall thickness is computed for respective CTA volumes, the next step is to remove the wall of the segmented tree. 


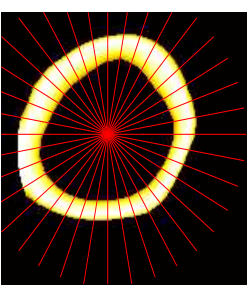

(a) Normal

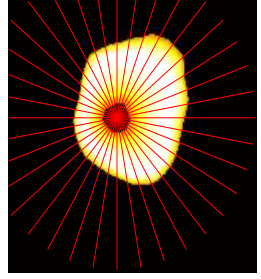

(b) Abnormal

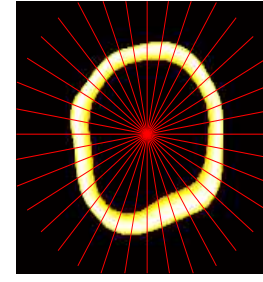

(c) Normal

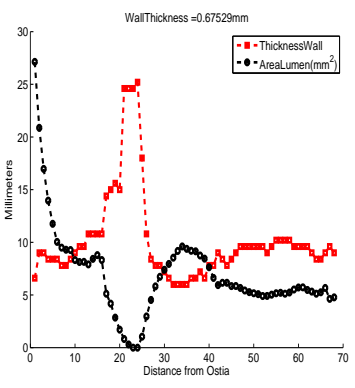

(d) Lumen area versus wall thickness plot

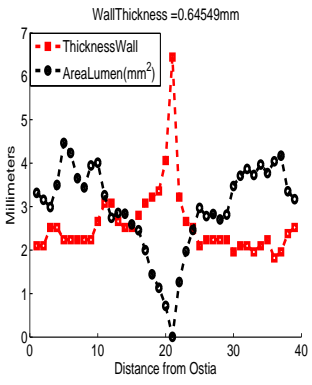

(e) Lumen area versus wall thickness plot
Fig. 6. Computation of the Vessel Wall thickness for coronary segment DS4 seg1. (a-c) shows the ray-projection to compute the mean thickness of the vessel wall, (d) represents the graphical comparison between lumen area and the normalized vessel wall thickness to reflect the anomalous lesion area. Cross sectional representing normal segment (a and c) leads to stable vessel wall, whereas abnormal cross section leads to expansion of the vessel wall based on low density soft plaques.

\section{PiXel-BASED Segmentation}

After removing the vessel wall from the segmented tree, it is expected that the leftover is true lumen and the noncalcified plaque (if any). Accordingly, we derive hand crafted discriminative features capable of differentiating voxels into lumen or non-calcified plaque. For voxel-wise discriminative features, we employed the spatial neighbourhood information, optimized 2-class GMM based posteriors, signed distance function, distance from the arterial orifice, pixel distance from the medial axis and histogram based fuzzy label as explained in this section.

\section{2-Class POSTERIORS}

It is notable that the non-calcified plaques present inside coronary vasculature do not follow any particular shape or structure; hence, the use of shape-prior information is not very effective in the problem domain. Consequently, the extensively investigated feature in context of non-calcified plaque segmentation is the intensity distribution in the vessel, as the plaque region undergoes an unexpected intensity drop relative to the normal blood HU distribution. Accordingly, we computed the intensity histogram for the plaque affected region with an expectation of two peaks representing the plaque and lumen respectively, as illustrated in Fig. 7a.

Next, the bi-modal intensity histogram of the plaque affected section is approximated using 2-class Gaussian Mixture Model, followed with the application of expectation maximization (EM) algorithm for optimal representation of two classes. Fig. 7b shows GMM approximation, with first class

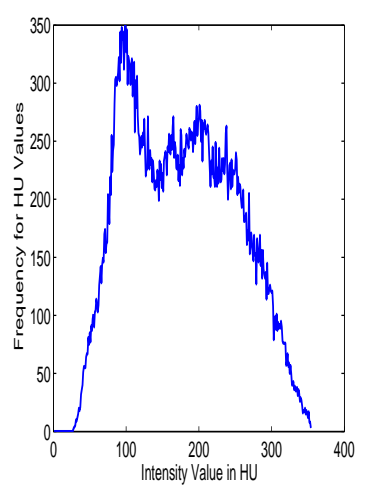

(a) Intensity histogram

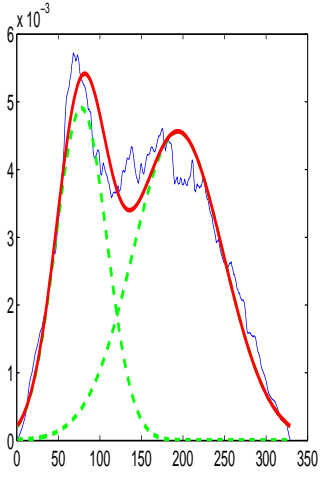

(b) 2-class GMM
Fig. 7. 2-class approximation for the plaque effected section of the coronary segment DS4 seg1. (a-b) shows the plaque effected boundary and respective bi-modal intensity histogram, (c) represents 2-class Gaussian Mixture Model and respective $\mathrm{HU}$ intensity peaks.

defining low density non-calcified plaque and the second class representing high intensity blood lumen.

After obtaining EM based optimal distribution parameters, we used Bayesian modelling approach to compute the posterior probabilities for two classes respectively as represented in Fig. 8 .

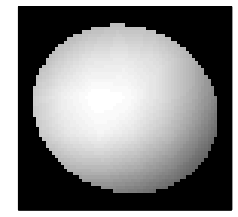

(a) Ideal vessel slice

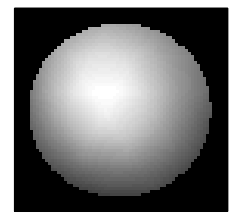

(d) Ideal vessel slice

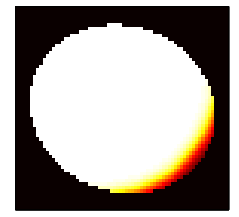

(b) GMM based Lumen

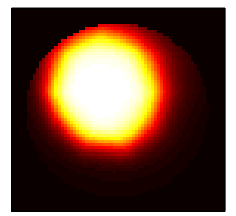

(e) GMM based lumen

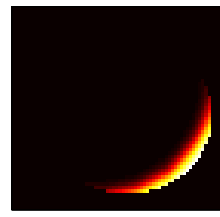

(c) GMM based Plaque

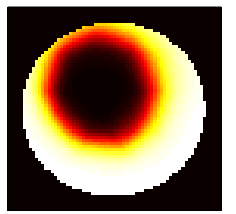

(f) GMM based plaque
Fig. 8. 2-class approximation based initial estimation for lumen and plaque Top row represents a normal cross-section, i.e. at the immediate start of the non-calcified plaque region, and second row represents cross-section in the mid of plaque region. Left column shows a 2D intensity based cross-section of the coronary vessel, whereas middle and right columns respectively shows the 2-class GMM based lumen and non-calcified plaque.

The left column represents the cross sectional view for an ideal vessel (plaque free vessel), the middle and right columns represents 2-class GMM based lumen and the plaque voxels, respectively. It can be observed that top row (start of the plaque) shows the 2-class lumen much close to the ideal vessel with a minimal plaque, however second row reflecting the mid of the plaque region shows significantly reduced lumen along with an expended plaque. Moreover, the relative position of the lumen and plaque validates the clinical fact that non-calcified plaque generally sticks with the vessel walls leading to Napkin 
ring signs [33].

From a statistical point of view, the computed plaque is compared against expert-based manual demarcations. Accordingly,we employed metrics true positive (TP), true negative (TN), false positive (FP), false negative(FN), respectively. True positive refers the case when expert-based lumen voxel is identified as lumen by posterior method. Similarly, True negative refers the case when expert-based plaque voxel is identified as plaque by the posterior method. In contrast, false positive and false negative defines two cases for mismatch among the expert-based lumen/plaque demarcation and the output of the posterior method. Using individual metrics, we computed Jaccard similarity index as follows.

$$
\text { Jaccardindex }=\frac{T P}{(T P+F P+F N)}
$$

It is important to mention that for an ideal plaque quantification, the Jaccard index approaches to one, whereas two dissimilar annotations result in Jaccard score of zero. According to the experimental results, mean Jaccard overlap is around $88 \%$ with respect to the manual expert. In terms of sensitivity, specificity and accuracy, the proposed methods achieves $84.13 \%, 79.15 \%$ and $82.02 \%$ success.

\section{CONClusion}

In this work, we proposed a method for voxel-wise quantification of coronary non-calcified plaque using Bayesian Posterior probability model. Based on the normal coronary segments, we computed the vessel-wall thickness in first step. In the subsequent step, we removed vessel wall from the segmented tree and employed Gaussian Mixture Model to compute intensity based clusters. According to the experimental results, it is shown that the automated plaque segmentation method achieves accuracy equivalent to human experts. We aim to extend this work in future in context of deep learning based solutions for the said problem. Application of convolutional neural network (CNN) shows promising results in recent years; however, this requires a bulk amount of data for adequate training.

\section{ACKNOWLEDGMENT}

We acknowledge the support of Dr. Ronak Rajani, consultant cardiologist, Guys \& St. Thomas Hospital, London and Dr. Pal Maurovich Horvat of Semmelweis University Hungary for the Clinical CTA data and ground truth references.

\section{REFERENCES}

[1] World Health Organization, Cardiovascular diseases CVDs, the global statistics, Available at http://www.who.int/mediacentre/factsheets/ fs $317 /$ en/(2016/11/11).

[2] U. K. NHS, Coronary Heart Disease, statistics for united kingdom (2016). URL http://www.nhs.uk/Conditions/Coronary-heart-disease/ Pages/Introduction.aspx

[3] T. Flohr, B. Ohnesorge, Multi-slice ct technology, in: Multi-slice and Dualsource CT in Cardiac Imaging, Springer, 2007, pp. 41-69.

[4] R. Virmani, A. P. Burke, A. Farb, F. D. Kolodgie, Pathology of the vulnerable plaque, Journal of the American College of Cardiology 47 (8s1) (2006) C13-C18.
[5] S. C. Saur, H. Alkadhi, L. Desbiolles, G. Szekely, P. C. Cattin, Automatic detection of calcified coronary plaques in computed tomography data sets, in: International Conference on Medical Image Computing and Computer- Assisted Intervention, Springer, 2008, pp. 170-177.

[6] G. Brunner, U. Kurkure, D. R. Chittajallu, R. P. Yalamanchili, I. A. Kakadiaris, Toward unsupervised classification of calcified arterial lesions, in: International Conference on Medical Image Computing and Computer- Assisted Intervention, Springer, 2008, pp. 144-152.

[7] I. Isgum, A. Rutten, M. Prokop, B. van Ginneken, Detection of coronary calcifications from computed tomography scans for automated risk assessment of coronary artery disease, Medical physics 34 (4) (2007) $1450-1461$

[8] B. Mohr, S. Masood, C. Plakas, Accurate lumen segmentation and stenosis detection and quantification in coronary cta, in: Proceedings of 3D Cardiovascular Imaging: a MICCAI segmentation challenge workshop, 2012.

[9] M. E. Clouse, A. Sabir, C.-S. Yam, N. Yoshimura, S. Lin, F. Welty, P. Martinez-Clark, V. Raptopoulos, Measuring noncalcified coronary atherosclerotic plaque using voxel analysis with mdct angiography: a pilot clinical study, American Journal of Roentgenology 190 (6) (2008) 1553- 1560.

[10] J. Wei, C. Zhou, H.-P. Chan, A. Chughtai, P. Agarwal, J. Kuriakose, L. Hadjiiski, S. Patel, E. Kazerooni, Computerized detection of noncalcified plaques in coronary ct angiography: Evaluation of topological soft gradient prescreening method and luminal analysis, Medical physics 41 (8) (2014) 081901.

[11] S. Lankton, A. Stillman, P. Raggi, A. Tannenbaum, Soft plaque detection and automatic vessel segmentation, in: 12th International Conference on Medical Image Computing and Computer Assisted Intervention (MICCAI), Springer Berlin Heidelberg, 2009, pp. 25-33.

[12] ] M. Tessmann, F. Vega-Higuera, D. Fritz, M. Scheuering, G. Greiner, Multiscale feature extraction for learning-based classification of coronary artery stenosis, in: SPIE Medical Imaging, International Society for Optics and Photonics, 2009, pp. 726002-726002.

[13] M. A. Zuluaga, I. E. Magnin, M. H. Hoyos, E. J. D. Leyton, F. Lozano, M. Orkisz, Automatic detection of abnormal vascular crosssections based on density level detection and support vector machines, International Journal of Computer Assisted Radiology and Surgery 6 (2) (2011) 163-174.

[14] F. Renard, Y. Yang, Image analysis for detection of coronary artery soft plaques in mdct images, in: 2008 5th IEEE International Symposium on Biomedical Imaging: From Nano to Macro, IEEE, 2008, pp. 25-28.

[15] Y. Li, W. Chen, K. Liu, Y.Wu, Y. Chen, C. Chu, B. Fang, L. Tan, S. Zhang, A voxel-map quantitative analysis approach for atherosclerotic noncalcified plaques of the coronary artery tree, Computational and mathematical methods in medicine 2013.

[16] S. Achenbach, F. Moselewski, D. Ropers, M. Ferencik, U. Homann, B. MacNeill, K. Pohle, U. Baum, K. Anders, I.-k. Jang, et al., Detection of calcified and noncalcified coronary atherosclerotic plaque by contrastenhanced, submillimeter multidetector spiral computed tomography, Circulation, 109 (1) (2004) 14-17.

[17] A. W. Leber, A. Becker, A. Knez, F. von Ziegler, M. Sirol, K. Nikolaou, B. Ohnesorge, Z. A. Fayad, C. R. Becker, M. Reiser, et al., Accuracy of 64-slice computed tomography to classify and quantify plaque volumes in the proximal coronary system: a comparative study using intravascular ultrasound, Journal of the American College of Cardiology 47 (3) (2006) 672-677.

[18] T. Schepis, M. Marwan, T. Pederer, M. Seltmann, D. Ropers, W. G. Daniel, S. Achenbach, Quantification of non-calcified coronary atherosclerotic plaques with dual-source computed tomography: comparison with intravascular ultrasound, Heart 96 (8) (2010) 610-615.

[19] Z. Sun, L. Xu, Coronary ct angiography in the quantitative assessment of coronary plaques, BioMed research international 2014.

[20] P. Schoenhagen, E. M. Tuzcu, A. E. Stillman, D. J. Moliterno, S. S. Hal liburton, S. A. Kuzmiak, J. M. Kasper, W. A. Magyar, M. L. Lieber, S. E. Nissen, et al., Non-invasive assessment of plaque morphology and remodeling in mildly stenotic coronary segments: comparison of 16-slice computed tomography and intravascular ultrasound, Coronary artery disease 14 (6) (2003) 459-462. 
[21] S. Schroeder, A. F. Kopp, A. Baumbach, C. Meisner, A. Kuettner, C. Georg, B. Ohnesorge, C. Herdeg, C. D. Claussen, K. R. Karsch, Noninvasive detection and evaluation of atherosclerotic coronary plaques with multislice computed tomography, Journal of the American College of Cardiology 37 (5) (2001) 1430-1435.

[22] ] H. Brodoefel, C. Burgstahler, M. Heuschmid, A. Reimann, F. Khosa, A. Kopp, S. Schroeder, C. Claussen, M. Clouse, Accuracy of dualsource ct in the characterisation of non-calcified plaque: use of a colourcoded analysis compared with virtual histology intravascular ultrasound, The British journal of radiology 82 (982) (2009) 805-812.

[23] D. Dey, T. Schepis, M. Marwan, P. J. Slomka, D. S. Berman, S. Achenbach, Automated three-dimensional quantification of noncalcified coronary plaque from coronary CT angiography: comparison with intravascular us, Radiology 257 (2) (2010) 516-522.

[24] W. Theo, The Great Challenge,, coronary artery stenoses detection and quantification evaluation framework (2016). URL http://www.http://coronary.bigr.nl/stenoses/

[25] H. Kirisli, M. Schaap, C. Metz, A. Dharampal, W. B. Meijboom, S.L. Papadopoulou, A. Dedic, K. Nieman, M. De Graaf, M. Meijs, et al., Standard ized evaluation framework for evaluating coronary artery stenosis detection, stenosis quantification and lumen segmentation algorithms in computed tomography angiography, Medical image analysis 17 (8) (2013) 859-876.

[26] M. M. Jawaid, R. Rajani, P. Liatsis, C. C. Reyes-Aldasoro, G. Slabaugh, A hybrid energy model for region based curve evolution-application to cta coronary segmentation, Computer Methods and Programs in Biomedicine 144C (2017) 189-202.

[27] ] M. M. Jawaid, A. Riaz, R. Rajani, C. C. Reyes-Aldasoro, G. Slabaugh, Framework for detection and localization of coronary non-calcified plaques in cardiac cta using mean radial profiles, Computers in Biology and 385 Medicine 89 (C) (2017) 84-95.

[28] ] W. C. Wong, A. C. Chung, Augmented vessels for quantitative analysis of vascular abnormalities and endovascular treatment planning, IEEE transactions on medical imaging 25 (6) (2006) 665-684.

[29] D.G. Kang, D. C. Suh, J. B. Ra, Three-dimensional blood vessel quantifi cation via centerline deformation, IEEE Transactions on Medical Imaging 28 (3) (2009) 405-414.
[30] A. F. Frangi, W. J. Niessen, R. M. Hoogeveen, T. Van Walsum, M. A. Viergever, Model-based quantitation of 3-d magnetic resonance angiographic images, IEEE Transactions on medical imaging 18 (10) (1999) 946-956.

[31] A. F. Frangi, W. J. Niessen, P. J. Nederkoorn, J. Bakker, W. P. T. M. Mali, M. A. Viergever, Quantitative analysis of vascular morphology from 3d MR angiograms: in vitro and in vivo results, Magnetic Resonance in Medicine 45 (2) (2001) 311-322.

[32] ] P. J. Yim, J. J. Cebral, R. Mullick, H. B. Marcos, P. L. Choyke, Vessel surface reconstruction with a tubular deformable model, IEEE transactions on medical imaging 20 (12) (2001) 1411-1421.

[33] N. R. Pal, S. K. Pal, A review on image segmentation techniques, Pattern recognition 26 (9) (1993) 1277-1294.

[34] O. MastO, B.Nico et al., Quantification of coronary plaque by 64-slice computed tomography: a comparison with quantitative intracoronary ultrasound, Investigative radiology, 43(9), (2008), 314-321.

[35] L. Athanasiou, G. Rigas, A.I. Sakellarios, T.P.Exarchos et al., Threedimensional reconstruction of coronary arteries and plaque morphology using CT angiographycomparison and registration with IVUS, BMC medical imaging, 16(1), (2016), p.9.

[36] T. Pflederer, M. Schmid, D. Ropers, U. Ropers, S. Komatsu, W. G. Daniel, and S. Achenbach, Interobserver variability of 64-slice computed tomography for the quantification of non-calcified coronary atherosclerotic plaque. RFo-Fortschritte auf dem Gebiet der Rontgenstrahlen und der bildgebenden Verfahren 179(9), 953-957.

[37] C. L. Christopher et al., How to assess non-calcified plaque in CT angiography: delineation methods affect diagnostic accuracy of lowattenuation plaque by CT for lipid-core plaque in histology, European Heart JournalCardiovascular Imaging 14(11), (2013): 1099-1105.

[38] D. Damini, et al., Automated 3-dimensional quantification of noncalcified and calcified coronary plaque from coronary CT angiography, Journal of cardiovascular computed tomography 3(6), (2009), 372-382.

[39] H. Brodoefel, C. Burgstahler, A. Sabir, C.S.Yam, F. Khosa, et al, Coronary plaque quantification by voxel analysis: dual-source MDCT angiography versus intravascular sonography. American Journal of Roentgenology, 192(3), (2009), W84-W89. 